\title{
28 Research Square \\ Intestinal Metabolomics of Juvenile Lenok \\ (Brachymystax Lenok) in Response to Heat Stress
}

\section{Yan Chen}

Beijing Academy of Agriculture and Forestry Sciences

\section{Yang Liu}

Qinghai University

Yucen Bai ( $\boldsymbol{\sim}$ baiyucen@foxmail.com )

China rural technology development center

Shaogang Xu

Beijing Academy of Agriculture and Forestry Sciences

\section{Xiaofei Yang}

Beijing Academy of Agriculture and Forestry Sciences

\section{Bo Cheng}

Chinese Academy of Fishery Sciences

\section{Research Article}

Keywords: heat stress, intestinal, metabolomics

Posted Date: November 15th, 2021

DOI: https://doi.org/10.21203/rs.3.rs-1030478/v1

License: (c) (1) This work is licensed under a Creative Commons Attribution 4.0 International License.

Read Full License 


\section{Abstract}

Changes in the metabolic profile within the intestine of lenok (Brachymystax lenok) when challenged to acute and lethal heat stress (HS) are studied using no-target HPLC-MS/MS metabonomic analysis. Of 51 differentially expressed metabolites identified in response to HS, 34 occurred in the positive ion mode and 17 in negative ion mode (VIP $>1, P<0.05)$. Changes in metabolites (i.e. alpha-D-glucose, stachyose and L-lactate) related to carbohydrate and glycolysis are identified in HS-treated lenok. Fatty acid $\beta$-oxidation in HS-treated lenok was inhibited by accumulation of acetyl carnitine, palmitoylcarnitine, carnitine, and erucic acid. Many amino acids (L-tryptophan, D-proline, L-leucine, L-phenylalanine, L-aspartate, L-tyrosine, L-methionine, L-histidine and L-glutamine) decreased to support energy demands in HS-treated lenok. Oxidative damage in HS-treated lenok was indicated by decreased glycerophospholipid metabolites (i.e. glycerophosphocholine, 1-palmitoyl-2-hydroxy-sn-glycero-3-phosphoethanolamine, 1-palmitoyl-sn-glycero3-phosphocholine, 1-stearoyl-2-oleoyl-sn-glycero-3-phosphocholine, and 1, 2-dioleoyl-sn-glycero-3phosphatidylcholine), and increased oxylipin production (12-HETE and 9R, 10S-EpOME). Oxidative stress increased formation of eicosanoids and dicarboxylic acids, overwhelming the mitochondrial $\beta$-oxidation pathway, while minor oxidative pathways (omega-oxidation and peroxisomal beta-oxidation) were likely to be activated in HS-treated lenok.

\section{Introduction}

Extreme and abrupt environmental changes are increasingly likely as a result of climate change. Not only have global temperature and precipitation patterns changed markedly in recent decades, but further change is predicted (Kibler et al., 2015; Yeo and Kim, 2014). Increased climate variability and extreme high temperature events at regional scales have impacted aquatic ecosystems, especially those of freshwater fish (Cqza et al., 2019; Newton et al., 2012; Clark et al., 2008; Susan et al., 2003). While fish can generally adapt to ranges of water temperature, acute and extreme fluctuations that exceed levels of tolerance will trigger series of stress-related responses, such as abnormal behavior, physiological dysfunction, biochemical reactions, and potentially death (Chen et al., 2021; Xia et al., 2017; Lu et al., 2016; Thorne et al., 2010).

The lenok (Brachymystax lenok) is a landlocked freshwater salmonid that has an extremely restricted distribution and small population size, occurs in upstream regions of cold rivers in East Asia (Liu et al., 2018). Although both artificial reproduction and breeding have been attempted for this species, natural lenok populations have decreased significantly with habitat degradation, and the species is now regarded to be endangered in Korea and China (Liu et al., 2018; Xu et al., 2014). Because lenok is very sensitive to fluctuation in water temperature, widespread declines in its populations may be a consequence of warming temperatures. Juvenile lenok function normally between 6 and $18^{\circ} \mathrm{C}$, but if climate events (e.g., high temperatures and droughts) in northern China become increasingly common and more extreme during summer, both cultured and wild fish will be affected (Liu et al., 2018; Mou et al., 2011). It is therefore important to understand how lenok respond to heat stress (HS) to mitigate the effects of these temperature extremes on its survival, and to maintain viable populations throughout its current 
distribution. Because this endangered species has a great impact on ecological diversity in its habitat, our research is of both practical and theoretical value for providing information about meeting climate change.

Systems biology approaches have been used to understand biological processes and metabolic changes in different tissues of cold water fishes following exposure to high temperature. Transcriptomics of the head kidney of rainbow trout Oncorhynchus mykiss in response to HS (between $18^{\circ} \mathrm{C}$ and $24^{\circ} \mathrm{C}$, increased $1^{\circ} \mathrm{C}$ per $24 \mathrm{~h}$ ) revealed modulated pathways in the immune system, protein metabolism, and the spliceosome (Huang et al., 2018). Under acute HS $\left(18-25^{\circ} \mathrm{C}\right.$, increased by $\left.2.5^{\circ} \mathrm{C} \mathrm{h}^{-1}\right)$, label-free quantification of protein expression in the rainbow trout liver changed in the estrogen signaling and platelet activation pathways, and complement and coagulation cascades (Kang et al., 2019). Regulation of DNA damage was reported in Chinook Salmon Oncorhynchus tshawytscha gill tissue after acute elevated temperature challenges $\left(14-21^{\circ} \mathrm{C}\right.$, increased by $\left.4^{\circ} \mathrm{C} \mathrm{h}^{-1}\right)$ (Clark et al., 2008). Therefore, it is apparent that HS induces tissue-specific responses in fish, and in these examples, salmonids.

Metabolomics is a basic discipline in systems biology, as are genomics, transcriptomics, and proteomics. Metabolomics approaches present new methods to study small endogenous metabolites and reveal changes in metabolites and metabolic pathway processes in response to external stimuli or disturbance. This technology has been widely used in disciplines of ecology, medicine and toxicology, and the biomarkers of metabolism or disease occur in complex organisms (Maha et al., 2019; Sun et al., 2018; Lardon et al., 2013). Non-targeted metabolomics is used to detect non-specific groups of metabolites in tissue samples, and has been applied in studies of metabolic changes in salmonids in response to HS challenges. Nuclear magnetic resonance (NMR)-based metabolomics has been conducted on plasma of Atlantic salmon (Oncorhynchus spp.) to reveal reprograming of amino acids, and energy and lipid metabolism following long-term (3 month) experimentation at high $\left(18^{\circ} \mathrm{C}\right)$ temperature (Kullgren et al., 2013). Liu (2018) identified thermal stress-activated glutamate metabolism in lenok liver tissue and plasma using an NMR-based metabonomic strategy, and suggested that glutamate might be a biomarker associated with moderate thermal stress $\left(24^{\circ} \mathrm{C}\right.$ for $7 \mathrm{~d}$ ) (Liu et al., 2018). To our knowledge no study has investigated acute responses to HS on the intestinal metabolome of lenok. We do so, and suggest ways to improve survival of this endangered species at extreme high temperature.

\section{Materials And Methods}

\section{Experimental design and sampling}

Healthy lenok were obtained from the Yanqing hatchery, Beijing Academy of Agriculture and Forestry Sciences, Institute of Fisheries Research (Beijing, China). Fish were first acclimated for 1 week at a mean temperature of $14 \pm 0.5^{\circ} \mathrm{C}, \mathrm{pH} 7.73 \pm 0.03$, and dissolved oxygen $7.58-8.55 \mathrm{mg} \mathrm{L}^{-1}$, with a light/dark photoperiod of $12: 12 \mathrm{~h}$. Following acclimation, 180 juvenile lenok $(23.5 \pm 2.64 \mathrm{~g}$ in body weight and 13.2 $\pm 0.59 \mathrm{~cm}$ in body length) were randomly selected and divided into two treatments (control (CT) and HS), each containing 90 fish (with no significant difference in body weight). For each treatment there were 3 
replicates, each containing 30 fish; fish were placed into rectangular tanks and further acclimated for 3 days. During acclimation fish were fed a commercial feed of $2 \%$ of their body weight twice a day ( 8 am, 4 $\mathrm{pm})$.

Our previous studies demonstrated that the semilethal high temperature of lenok at $48 \mathrm{~h}$ was $26.3^{\circ} \mathrm{C}$. Water temperature in the $\mathrm{CT}$ treatment was maintained at $14^{\circ} \mathrm{C}$, whereas that for the HS treatment was gradually increased from $14^{\circ} \mathrm{C}$ to $26^{\circ} \mathrm{C}$ at $1^{\circ} \mathrm{C} \mathrm{h}^{-1}$, then maintained at $26^{\circ} \mathrm{C}$ for $48 \mathrm{~h}$. No food was provided to fish during heat stress treatment. Fish were considered dead if immobile and non-responsive when probed with a glass rod. Dead fish were recorded and removed immediately.

After HS experimentation, nine fish (three from each replicate tank) from each treatment were collected and euthanized with a solution containing $\sim 250 \mathrm{mg} \mathrm{L}^{-1}$ ethyl 3-aminobenzoate methane sulfonate (MS222; TCI, Tokyo, Japan). Intestinal tissues (without feces) were immediately removed from each fish, and intestinal samples were stored separately in $1.5 \mathrm{~mL}$ centrifuge tubes at $-80^{\circ} \mathrm{C}$ until metabolomics analyses.

Intestinal metabolomics analysis

Intestinal samples were homogenized. Metabolite extraction was performed using methanol and acetonitrile (volume ratio 1:1), with $20 \mu \mathrm{l}$ of each sample taken for quality control, and the rest for LC-MS detection. Analyses were performed using an UHPLC (1290 infinity LC, Agilent Technologies) system coupled to a quadrupole time-of-flight (AB Sciex TripleTOF 6600) system at the Shanghai Applied Protein Technology Co., Ltd. The HILIC separation was accomplished using an ACQUITY UPLC BEH $(2.1 \mathrm{~mm}$. $100 \mathrm{~mm}, 1.7 \mu \mathrm{m}$, water, Ireland) column. LC-MS/MS analysis was performed on a Q Exactive mass spectrometer (Thermo Scientific). Data-dependent acquisition MS/MS experiments were performed with HCD scans. Dynamic exclusion was implemented to remove some unnecessary information in the MS/MS spectra. Mass spectrometry was operated in both positive and negative ion modes.

\section{Data processing}

Raw MS data (wiff.scan files) were converted to mzXML files using ProteoWizard msConvert and processed using XCMS for feature detection, retention time correction, and peak alignment. In extracted ion features, only variables with $>50 \%$ nonzero measurement values in at least one group were kept. Compound identification of metabolites was performed by comparing the accuracy of $\mathrm{m} / \mathrm{z}$ values $(<25$ $\mathrm{ppm}$ ), retention time, molecular weight, secondary fragmentation spectrum, collision energy, and other information of the MS/MS spectra with the standard product (in-house database) built by Shanghai Applied Protein Technology. Results were checked and confirmed manually, to ensure that identification was at or better than structural level 2. All identified metabolites (combined positive and negative ion modes) were classified and counted according to their chemical classification information.

Data statistical analysis 
The R package DEP 1.5.1 was used for statistical analyses of all metabolomics data (all metabolites, including unidentified ones, in both positive and negative ion modes). Data are expressed as log2 (fold change) ( $\left.\log _{2} \mathrm{FC}\right)$ compared with control samples. Metabolites with $\mathrm{FC}>1.5$ or $\mathrm{FC}<0.67$ and $\mathrm{P}$ values less than 0.05 applied to Student's t-test are graphed in volcano plots.

After normalizing to total peak intensity, processed data were analyzed using R. Multivariable data analyses (Pareto-scaled principal component analysis (PCA), and orthogonal partial least-squares discriminant analysis (OPLS-DA)) were performed. PCA was performed to show the distribution of origin data. OPLS-DA was applied to obtain a high level of group separation, and an understanding of variables responsible for a classification. A 7-fold cross validation and response permutation test were conducted to estimate model robustness. Variable importance in projection (VIP) values for each variable in the OPLS-DA model were calculated to indicate their contribution to the classification. Metabolites with VIP values $>1$ and $P$ values $<0.05$ that were applied to Student's $t$-test at univariate level between the treatments were considered statistically significant.

Bioinformatic analysis of differentially expressed metabolites

To more comprehensively and intuitively display the metabolite expression patterns, data for the relative expression of metabolites were used to perform hierarchical clustering analysis using Cluster3.0 (http://bonsai.hgc.jp/ mdehoon/software/cluster/software.htm). A heat map is presented as a visual aid to visualize the differential metabolites of lenok in response to heat stress.

Metabolites were blasted against the online Kyoto Encyclopedia of Genes and Genomes (KEGG) database (http://geneontology.org/) to retrieve COs, and were subsequently mapped to pathways in KEGG. Corresponding KEGG pathways were extracted. The metabolic network of metabolites that differed significantly between CT and HS treatments was profiled based on KEGG annotation information and biological function.

\section{Results}

No death occurred in control group, and the morality of lenok was $57 \pm 15 \%$ in HS group after 48 h heat stress.

Metabolite identification

The 396 metabolites included 333 identified in positive ion mode and 106 in negative ion mode. All metabolites were classified and counted according to their Chemical Taxonomy (Figure 1). The number of metabolites in the following super classes were: organic acids and derivatives (59); lipids and lipid-like molecules (29); nucleosides, nucleotides and analogs (25); organoheterocyclic compounds (25); organic oxygen compounds (24); organic nitrogen compounds (11); benzenoids (9); phenylpropanoids and polyketides (7); nucleosides, nucleotides, and analogs/organ heterocyclic compounds (1); organosulfur compounds (1); and undefined (205). 
Figure 1

Differentially expressed metabolites (including unidentified ones) detected in both positive and negative ion modes were analyzed based on univariate analysis. Metabolites with FC $>1.5$ or FC $<0.67$, and $p<$ 0.05 were visualized in volcano plots (Figure 2).

Figure 2

PCA and OPLS-DA analysis

PCA was performed to identify intrinsic pattern within the data set. PCA score plots are shown in Figure3. Model evaluation parameters obtained after 7-fold cross validation were $R^{2} X=0.571$ in the positive ion mode (Fig. 3a) model, and $R^{2} X=0.592$ in the negative ion mode (Fig. 3b) model. The closer the $R^{2} X$ is to 1 , the more reliable a model.

Figure 3

The OPLS-DA model was prepared to obtain clear separation between the CT and HS treatments. Model evaluation parameters obtained after 7-fold cross validation for the positive ion mode were $R^{2} X=0.362$, $R^{2} Y=0.938$, and $Q^{2} Y=0.598$, and for negative ion mode $R^{2} X=0.452, R^{2} Y=0.934$, and $Q^{2} Y=0.706$. All parameters were stable and effective for fitness and prediction. A permutation test was used to verify the model to avoid overfitting of supervised models to ensure their effectiveness; $\mathrm{R}^{2}$ and $\mathrm{Q}^{2}$ intercept values determined after permutations were 0.804 and -0.336 (positive ion mode) and 0.644 and -0.449 (negative ion mode). Low $\mathrm{Q}^{2}$ intercept values indicate that the robustness of the models presented low overfitting and reliability risks. All samples in score plots were within a $95 \%$ confidence ellipse prepared using Hotelling"s T-squared, with clear separation and discrimination between pairwise groups. The OPLS-DA model identified differences between treatments and in subsequent analyses (Figure 4).

\section{Figure 4}

Differentially expressed metabolites

Based on the OPLS-DA and Student's t-test analyses, significantly different metabolites occurred in CT and HS treatments. Of 51 differentially expressed metabolites, 34 were identified in the positive ion mode and 17 in the negative ion mode (Table S1). Hierarchical clustering analysis was performed to visualize differences in the metabolome of these two treatments. Compared with the CT treatment, 8 metabolites in the HS treatment were up-regulated (red) and 26 metabolites were down-regulated (blue) in the positive ion mode, and 6 metabolites were up-regulated and 11 metabolites were down-regulated in the negative ion mode (Figure 5).

Figure 5 
To visualize the potential metabolic response of lenok to HS, the metabolic network of differentially expressed metabolites was built according to KEGG annotation information (Figure 6). The metabolites are colored according to the type of change after the heat stress. The up-regulated metabolites including $\mathrm{N}$-acetyl---alanine, 4-oxoretinol, 12-hete, L-palmitoylcarnitine, 9r, 10s-epome, acetyl carnitine, erucic acid, glutathione disulfide, creatine, lactate and l-carnitine were colored into red. The down-regulated metabolites including cytosine, D-proline, 1-stearoyl-2-oleoyl-sn-glycerol 3-phosphocholine (SOPC), 1,2dioleoyl-sn-glycero-3-phosphatidylcholine, L-tryptophan, taurocholate, L-aspartate, 1-palmitoyl-sn-glycero3-phosphocholine, 1-stearoyl-sn-glycerol 3-phosphocholine, L-leucine, uracil, cytidine, L-pyroglutamic acid, L-tyrosine, trans-cinnamate, alpha-D-glucose, dopamine, adenosine 3'-monophosphate, L-phenylalanine, $\mathrm{N}$-acetylmannosamine, L-histidine, L-glutamine, L-methionine, stachyose, glycerophosphocholine, 1Palmitoyl-2-hydroxy-sn-glycero-3-phosphoethanolamine, 2-hydroxyadenine, taurochenodeoxycholate and adenosine were colored into green.

Figure 6

\section{Discussion}

As poikilotherms, the effects of temperature on fish can be profound, because their body temperature changes with that of ambient water (Mueller et al., 2015; Machado et al., 2014; Windisch et al., 2014; Scott and Johnston, 2012). Since the 1990s predictions have been made about the increased frequency and severity of climate events in the future, and for them to occur for longer (Ikeda et al., 2012). The optimal temperature for lenok growth is between $14^{\circ} \mathrm{C}$ and $18^{\circ} \mathrm{C}$ (Liu et al., 2011), but they may experience higher temperatures during seasonal changes and heat currents in more southern habitats. We evaluated the effect of HS on changes in the metabolic profile within the intestine of lenok using notarget HPLC-MS/MS metabonomic analysis.

Metabolic regulation is an important strategy by which fish adapt to environmental stress (Melvin et al., 2018; Martyniuk and Simmons, 2016). Metabolomic analysis provides an integrated description of HSinduced metabolic changes in the intestine of lenok, and enables identification of differentially expressed metabolites resulting from acute HS. Changes in metabolites (i.e. alpha-D-glucose, stachyose, and Llactate) related to carbohydrate and energy metabolism (Fig. 6) suggest that HS strongly influences energy pathways. Decreased glucose and stachyose stores in the intestine of HS-treated lenok indicated increased energy expenditure with increased temperature. Stimulation of glycogenosis had been reported in several fish species when exposed to extreme high temperature to meet energy demands (Forgati et al., 2017; Xia et al., 2017; Lu et al., 2016), resulting in decreased glucose and carbohydrate levels. Lactate, a major end-product of anaerobic metabolism (Lu et al., 2016), accumulated in the intestine of HS-treated lenok. In conjunction with decreased glucose, lenok increased anaerobic metabolic activity under stress when exposed to heat. HS also reduced oxygen concentration and $\mathrm{PO}_{2}$ in fish; induced functional hypoxia could promote expression of hypoxia inducible factor 1 (HIF-1) to maintain oxygen homeostasis (Islam et al., 2020; Thomsen et al., 2017; Semenza, 2012). Fish resorted to anaerobic glycolysis to meet energy demands under these conditions. 
Differentially expressed metabolites (acetyl carnitine, palmitoylcarnitine, carnitine, 12-HETE, 9R, 10SEpOME, and erucic acid) appear to be linked to fatty acid metabolism. In animals, the carnitine pool comprises L-carnitine and acylcarnitine ester, which play important roles in mitochondrial $\beta$-oxidation of long-chain fatty acids and ATP production (Wang et al., 2016; Ozorio et al., 2010). Major physiological functions of carnitine involve transferal of long-chain fatty acids by conjugation of acyl residues to the $\beta$ hydroxyl group on the carnitine molecule. The carnitine derivative of the long-chain fatty (usually palmitoylcarnitine), formed by carnitine palmitoyltransferase-I (CPT-I) in the mitochondrial outer membrane, enters the mitochondrial matrix in exchange for a free carnitine (Sabzi et al., 2017; Neto et al., 2012; Ozorio et al., 2010). As the most common carnitine ester, acetyl-L-carnitine transports acetyl groups to different regions. Accumulation of $\mathrm{L}$-carnitine and the acylcarnitine ester may indicate inhibition of $\beta$ oxidation, as might accumulation of the unsaturated fatty (erucic) acid (Vetter et al., 2020; Sharma and Black, 2009). Unlike previous studies which demonstrated metabolic rate, including fatty acid metabolism, to be promoted to feed energy demand following increased environmental temperature, we report the energy source from fatty acid $\beta$-oxidation in HS-treated lenok to be inhibited (Chen et al., 2021; Hermann et al., 2019; Forgati et al., 2017; Thorne et al., 2010).

The expression of many amino acids (D-proline, and L-tryptophan, L-leucine, L-phenylalanine, L-aspartate, L-tyrosine, L-methionine, L-histidine and L-glutamine) decreased in HS-treated lenok. In fish, amino acids represent a major substratum for energy production (Li et al., 2020; Jia et al., 2017). With increasing energy loss in stress conditions, amino acids could function as an immediate source of fuel to produce energy to maintain pathway function (Lu et al., 2017; Li et al., 2009). Energy limitation accounted for large-scale decreases in amino acids in fish when energy metabolism was inhibited, because these pools of oxidizable amino acids were used extensively in energy metabolism in severe conditions (Maha et al., 2019; Kullgren et al., 2013). Additionally, adenosine is a basic component of synthesis energy substances, such as adenosine triphosphate (ATP), coenzyme nicotinamide adenine dinucleotide (NAD), and flavin adenine dinucleotide (FAD) (Duan et al., 2021; Baldissera et al., 2018). Cytidine, cytosine and uracil can be catalyzed by dihydropyrimidine dehydrogenase (DPD) to dihydrouracil, which could be used for synthesis of coenzyme A (CoA) and energy metabolism (Duan et al., 2021) (Figure 6). Decreases in adenosine, uracil, cytidine, and cytosine may further indicate energy loss and deficiency in HS-treated lenok.

Oxidative stress is a common response associated with acute heat stress in fish because of the imbalance between production of reactive oxygen species (ROS) and the capacity of ROS scavenging (Chen et al., 2021; Xia et al., 2017; Qian and Xue, 2016). Fish exposed to acute heat have increased lipid and protein oxidation markers, DNA damage, and cellular injury (Banh et al., 2016; Logan et al., 2011; Kaur et al., 2010). Oxidative damage in HS-treated lenok was indicated by decreased metabolites of glycerophospholipid (i.e. glycerophosphocholine, 1-palmitoyl-2-hydroxy-sn-glycero-3phosphoethanolamine, 1-palmitoyl-sn-glycero-3-phosphocholine, 1-stearoyl-2-oleoyl-sn-glycero-3phosphocholine, and 1,2-dioleoyl-sn-glycero-3-phosphatidylcholine), and increased oxylipin production (i.e. 12-HETE and 9R,10S-EpOME) (Figure 6). Glycerophospholipids are the main cell membrane lipids, and play important roles in, for example, regulating membrane potential, curvature, and ion transport 
(Zhang et al., 2020; Melvin et al., 2019; Shimura et al., 2016). Downregulation of glycerophospholipids along with oxidative stress in fish might indicate changes in the structure and function of cellular membranes (Melvin et al., 2019; van Meer et al., 2008). Hydroperoyeicosatetraenoic acids (HETEs) and epoxyoctadecamonoenoic acids (EpOMEs) derived from arachidonic acid (AA) and linoleic acid (LA), respectively, are associated with oxidative stress and pathological conditions in organisms, including fish (Hildreth et al., 2020; Zarini et al., 2014). AA, found in a bound (rather than free) form as a cell membrane phospholipid could be released by lipases like phospholipase A2 (PLA2) after disruption of the cell membrane. In the presence of a lipoxygenase (LOs)-mediated pathway, AA is oxidized into hydroperoyeicosatetraenoic acid (HETE) (Zarini et al., 2014; Yang et al., 2006; Rocha et al., 2003). Leukotoxin (9R, 10S-EpOME), an epoxide derivative of linoleic acid, is synthesized by cytochrome P450 monooxygenases (Vatanparast et al. 2020). Increased 12-HETE and 9R, 10S-EpOME in HS-treated fish, consistent with previous findings for fish under stress, reveals the activation of arachidonic acid released from membrane phospholipids and oxidative stress in HS-treated fish (Maha et al., 2019; Baldissera et al., 2018).

Transcriptomic responses to heat stress in gill and liver tissue of Brachymystax lenok tsinlingensis demonstrated that lethal heat stress triggered series dynamic metabolic changes of the heat shock and immune response, metabolic adjustment and ion transport (Li et al., 2021). The semi-lethal thermal stress to lenok demonstrated the metabolism changes including repression of energy metabolism, catabolism of amino acids, biosynthesis of glutamate and glutamine and increases in lipids in plasma according to NMR-based metabolomics (Liu et al., 2019). Our data provide a new insight of metabolism changes in intestine of lenok and suggest that oxidative stress increased formation of both eicosanoids and dicarboxylic acids, overwhelming the mitochondrial $\beta$-oxidation pathway, while minor oxidative pathways (omega-oxidation and peroxisomal beta-oxidation) were likely to be activated in HS-treated fish. Series of metabolic reactions might be induced in an attempt to reduce inflammatory tissue damage. Except for increased cellular glutathione (GSH) to scavenge ROS, L-carnitine and acylcarnitine ester played protective roles against oxidative stress in fish (Li et al., 2019; Wang et al., 2016; Guzman-Guillen et al., 2013). Accumulation of L-carnitine and acylcarnitine ester may act to inhibit aerobic oxidation of lipids to reduce indices of oxidative stress. As main components of phospholipids in cell membranes, decreases in glycerophospholipids could significantly affect the permeability and polarity of cell membranesregarded to be a defense mechanism to prevent ROS from entering cells and reducing oxidative stress (Zhang et al., 2020; Melvin et al., 2019).

\section{Declarations}

\section{Author contribution}

Yan Chen was responsible for experimental design and manuscript writing. Yang Liu took part in sampling and funding acquisition. Yucen Bai supervised the research project and supervised the writing of the manuscript. Xiaofei Yang took part in experimental procedures. Shaogang Xu was responsible for preliminary investigation, supervised the research project and funding acquisition. 
Funding

The work was fund support by Fund of Key Laboratory of Control of Quality and Safety for Aquatic Products, Ministry of Agriculture and Rural Affairs, P. R. China, Qinghai Science and Technology Department Project (No. 2018-ZJ-703), National Natural Science Foundation of China (No. 31760763) and Beijing technical industry system project (pxm2021 179303 000022).

Data availability

The data used to support the findings of this study are available from the corresponding author upon request.

\section{Ethics approval}

This study was approved by the Institutional Animal Care and Use Committee of Beijing Fisheries Research Institute, Beijing Academy of Agriculture and Forestry Sciences. All experimental procedures were in compliance with the guidelines of Yanqing site of the Beijing Academy of Agriculture and Forestry Sciences, Institute of Fisheries Research, Beijing, China.

Conflict of interest

The authors declare no competing interests.

\section{References}

Baldissera, M.D., Souza, C.F., Bottari, N.B., Verdi, C.M., Santos, R.C.V., Vizzotto, B.S., Baldisserotto, B., 2018. Purinergic signalling displays an anti-inflammatory profile in the spleen of fish experimentally infected with Aeromonas caviae: Modulation of the immune response. Journal of Fish Diseases 41, 683-687.

Banh, S., Wiens, L., Sotiri, E., Treberg, J.R., 2016. Mitochondrial reactive oxygen species production by fish muscle mitochondria: Potential role in acute heat-induced oxidative stress. Comparative Biochemistry and Physiology Part B: Biochemistry and Molecular Biology 191, 99-107.

Chen, Y., Liu, E., Li, C., Pan, C., Zhao, X., Wang, Y., Ling, Q., 2021. Effects of heat stress on histopathology, antioxidant enzymes, and transcriptomic profiles in gills of pikeperch Sander lucioperca. Aquaculture 534, 736277.

Clark, T.D., San Db Lom, E., Cox, G.K., Hinch, S.G., Farrell, A.P., 2008. Circulatory limits to oxygen supply during an acute temperature increase in the Chinook salmon (Oncorhynchus tshawytscha). Am J Physiol Regul Integr Comp Physiol 295, R1631-1639.

Cqza, B., Peng, Z.A., Ylr, A., Lhc, B., Jlw, A., 2019. Physiological response and miRNA-mRNA interaction analysis in the head kidney of rainbow trout exposed to acute heat stress. Journal of Thermal Biology 83, 134-141. 
Duan, Y., Xiong, D., Wang, Y., Li, H., Dong, H., Zhang, J., 2021. Toxic effects of ammonia and thermal stress on the intestinal microbiota and transcriptomic and metabolomic responses of Litopenaeus vannamei. Science of The Total Environment 754, 141867.

Forgati, M., Kandalski, P.K., Herrerias, T., Zaleski, T., Machado, C., Souza, M.R.D.P., Donatti, L., 2017. Effects of heat stress on the renal and branchial carbohydrate metabolism and antioxidant system of Antarctic fish. Journal of Comparative Physiology B 187, 1137-1154.

Guzman-Guillen, R., Prieto, A.I., Vazquez, C.M., Vasconcelos, V., Camean, A.M., 2013. The protective role of L-carnitine against cylindrospermopsin-induced oxidative stress in tilapia (Oreochromis niloticus). Aquatic Toxicology 132, 141-150.

Hermann, B.T., Wuertz, S., Vanselow, K.H., Schulz, C., Stiller, K.T., 2019. Divergent gene expression in the gills of juvenile turbot (Psetta maxima) exposed to chronic severe hypercapnia indicates dose-dependent increase in intracellular oxidative stress and hypoxia. Aquatic Toxicology 206, 72-80.

Hildreth, K., Kodani, S.D., Hammock, B.D., Zhao, L., 2020. Cytochrome P450-derived linoleic acid metabolites EpOMEs and DiHOMEs: a review of recent studies. The Journal of Nutritional Biochemistry $86,108484$.

Huang, J., Li, Y., Liu, Z., Kang, Y., Wang, J., 2018. Transcriptomic responses to heat stress in rainbow trout Oncorhynchus mykiss head kidney. Fish \& Shellfish Immunology 82, 32-40.

Ikeda, K., Prein, A., Rasmussen, R., Liu, C., Holland, G.J., 2012. Impact of climate change on heavy precipitation events: application of extreme value theory to a future climate simulation over the colorado headwaters region, AGU Fall Meeting Abstracts, 2012AGUFM.A41H0070I.

Islam, M.J., Slater, M.J., Kunzmann, A., 2020. What metabolic, osmotic and molecular stress responses tell us about extreme ambient heatwave impacts in fish at low salinities: The case of European seabass, Dicentrarchus labrax. Science of The Total Environment 749, 141458.

Jia, S., Li, X., Zheng, S., Wu, G., 2017. Amino acids are major energy substrates for tissues of hybrid striped bass and zebrafish. Amino Acids 49, 1-11.

Kang, Y., Liu, Z., Shi, H., Wang, J., Huang, J., Li, Y., Li, J., Wang, Y., 2019. Label-free quantification of protein expression in the rainbow trout (Oncorhynchus mykiss) in response to short-term exposure to heat stress. Comparative biochemistry and physiology. Part D, Genomics \& proteomics 30,158-168.

Kaur, M., Atif, F., Ali, M., Rehman, H., Raisuddin, S., 2010. Heat stress-induced alterations of antioxidants in the freshwater fish Channa punctata Bloch. Journal of Fish Biology 67, 1653-1665.

Kullgren, A., Jutfelt, F., Fontanillas, R., Sundell, K., Samuelsson, L., Wiklander, K., Kling, P., Koppe, W., Larsson, D.J., Björnsson, B.T., 2013. The impact of temperature on the metabolome and endocrine 
metabolic signals in Atlantic salmon (Salmo salar). Comparative Biochemistry and Physiology Part A: Molecular \& Integrative Physiology 164, 44-53.

Kibler, S.R., Tester, P.A., Kunkel, K.E., Moore, S.K., Litaker, R.W., 2015. Effects of ocean warming on growth and distribution of dinoflagellates associated with ciguatera fish poisoning in the Caribbean. Ecological Modelling 316, 194-210.

Lardon, I., Nilsson, G.E., Stecyk, J.A., Vu, T.N., Laukens, K., Dommisse, R., De Boeck, G., 2013. 1 H-NMR study of the metabolome of an exceptionally anoxia tolerant vertebrate, the crucian carp (Carassius carassius). Metabolomics 9, 311-323.

Li, J.M., Li, L.Y., Zhang, Y.X., Jiang, Z.Y., Limbu, S.M., Qiao, F., Degrace, P., Chen, L.Q., Zhang, M.L., Du, Z.Y., 2019. Functional differences between $\mathrm{I}$ - and d-carnitine in metabolic regulation evaluated using a lowcarnitine Nile tilapia model. British Journal of Nutrition 122, 625-638.

Li, P., Liu, Q., Li, J., Wang, F., Wen, S., N Li. 2021. Transcriptomic responses to heat stress in gill and liver of endangered brachymystax lenok tsinlingensis. Comparative Biochemistry and Physiology Part D Genomics and Proteomics, 38, 100791.

Li, P., Mai, K., Trushenski, J., Wu, G., 2009. New developments in fish amino acid nutrition: towards functional and environmentally oriented aquafeeds. Amino Acids 37, 43-53.

Li, X., Shixuan Zheng, Jia, S., Song, F., Zhou, C., Wu, G., 2020. Oxidation of energy substrates in tissues of largemouth bass (Micropterus salmoides). Amino Acids 52, 1017-1032.

Liu, Y., Liu, H., Xu, G., Mou, Z., Yin, J., 2018. Effects of water temperature on feeding and growth of the lenok Brachymystax lenok (Pallas) with different sizes. Journal of Fishery Sciences of China 25, 286-293.

Liu, Y., Mou, Z.B., Xu, G.F., Li, Y. F., 2011. Effects of water temperature on activities of digestive enzymes in juvenile lenok Brachymystax lenok. Chinese Journal of Fisheries 24,:6-9. (in Chinese with English abstract)

Logan, Cheryl, A., Somero, George, N., 2011. Effects of thermal acclimation on transcriptional responses to acute heat stress in the eurythermal fish Gillichthys mirabilis (Cooper). American Journal of Physiology: Regulatory, Integrative \& Comparative Physiology 300(6):R1373-83.

Lu, J., Shi, Y., Cai, S., Feng, J., 2017. Metabolic responses of Haliotis diversicolor to Vibrio parahaemolyticus infection. Fish \& Shellfish Immunology 60, 265-274.

Lu, Y., Wu, Z., Song, Z., Xiao, P., Liu, Y., Zhang, P., You, F., 2016. Insight into the heat resistance of fish via blood: Effects of heat stress on metabolism, oxidative stress and antioxidant response of olive flounder Paralichthys olivaceus and turbot Scophthalmus maximus. Fish \& shellfish immunology 58, 125-135. 
Machado, C., Zaleski, T., Rodrigues, E., dos Santos Carvalho, C., Cadena, S.M.S.C., Gozzi, G.J., Krebsbach, P., Rios, F.S.A., Donatti, L., 2014. Effect of temperature acclimation on the liver antioxidant defence system of the Antarctic nototheniids Notothenia coriiceps and Notothenia rossii. Comparative Biochemistry and Physiology Part B: Biochemistry and Molecular Biology 172, 21-28.

Maha, I.F., Xie, X., Zhou, S., Yu, Y., Liu, X., Zahid, A., Lei, Y., Ma, R., Yin, F., Qian, D., 2019. Skin metabolome reveals immune responses in yellow drum Nibea albiflora to Cryptocaryon irritans infection. Fish \& Shellfish Immunology 94, 661-674.

Martyniuk, C.J., Simmons, D.B., 2016. Spotlight on environmental omics and toxicology: a long way in a short time. Comparative Biochemistry and Physiology Part D: Genomics and Proteomics 19, 97-101.

Melvin, S.D., Lanctôt, C.M., Doriean, N.J., Carroll, A.R., Bennett, W.W., 2018. Untargeted NMR-based metabolomics for field-scale monitoring: Temporal reproducibility and biomarker discovery in mosquitofish (Gambusia holbrooki) from a metal (loid)-contaminated wetland. Environmental Pollution $243,1096-1105$.

Melvin, S.D., Lanctot, C.M., Doriean, N.J.C., Bennett, W., Carroll, A.R., 2019. NMR-based lipidomics of fish from a metal (loid) contaminated wetland show differences consistent with effects on cellular membranes and energy storage. Science of the Total Environment 654, 284-291.

Mou, Z., Liu, Y., Xu, G., Li, Y., 2011. The optimum temperature for growth and feeding in Brachymystax lenok. Chinese Journal of Fisheries 24, 6-8 (in Chinese with English abstract).

Mueller, C.A., Eme, J., Manzon, R.G., Somers, C.M., Boreham, D.R., Wilson, J.Y., 2015. Embryonic critical windows: changes in incubation temperature alter survival, hatchling phenotype, and cost of development in lake whitefish (Coregonus clupeaformis). Journal of Comparative Physiology B 185, 315331.

Neto, E.V., Fonseca, A.A., Almeida, R.F., Figueiredo, M.P., Porto, M., Ribeiro, M.G., 2012. Analysis of acylcarnitine profiles in umbilical cord blood and during the early neonatal period by electrospray ionization tandem mass spectrometry. Brazilian Journal of Medical \& Biological Research 45, 546-556.

Newton, J.R., Santis, C.D., Jerry, D.R., 2012. The gene expression response of the catadromous perciform barramundi Lates calcarifer to an acute heat stress. Journal of Fish Biology 81, 81-93.

Ozorio, R., Ginneken, V., Rui, J., Verstegen, M., Huisman, E.A., 2010. Effects of exercise on I-carnitine and lipid metabolism in African catfish (Clarias gariepinus) fed different dietary l-carnitine and lipid levels. British Journal of Nutrition 103, 1139-1150.

Qian, B., Xue, L., 2016. Liver transcriptome sequencing and de novo annotation of the large yellow croaker (Larimichthy crocea) under heat and cold stress. Marine Genomics 25, 95-102. 
Rocha, P.N., Plumb, T.J., Coffman, T.M., 2003. Eicosanoids: lipid mediators ofinflammation in transplantation. Springer Seminars inlmmunopathology 25, 215-227.

Sabzi, E., Mohammadiazarm, H., Salati, A.P., 2017. Effect of dietary l-carnitine and lipid levels on growth performance, blood biochemical parameters and antioxidant status in juvenile common carp (Cyprinus carpio). Aquaculture 480, 89-93.

Scott, G.R., Johnston, I.A., 2012. Temperature during embryonic development has persistent effects on thermal acclimation capacity in zebrafish. Proceedings of the National Academy of Sciences 109, 1424714252.

Semenza, G., 2012. Hypoxia-inducible factors in physiology and medicine. Cell 148, 399-408.

Sharma, S., Black, S.M., 2009. Carnitine homeostasis, mitochondrial function and cardiovascular disease. Drug Discovery Today: Disease Mechanisms 6, e31-e39.

Shimura, M., Shindou, H., Szyrwiel, L., Tokuoka, S.M., Hamano, F., Matsuyama, S., Okamoto, M., Matsunaga, A., Kita, Y., Ishizaka, Y., Yamauchi, K., Kohmura, Y., Lobinski, R., Shimizu, I., Shimizu, T., 2016. Imaging of intracellular fatty acids by scanning X-ray fluorescence microscopy. Faseb Journal 30, 41494158.

Sun, Y.C., Wu, S., Du, N.N., Song, Y., Xu, W., 2018. High-throughput metabolomics enables metabolite biomarkers and metabolic mechanism discovery of fish in response to alkalinity stress. RSC advances 8 , 14983-14990.

Susan, G. L., Mervyn, E.A., Bruce, L. T., 2003. Red blood cell Hsp 70 mRNA and protein as bio-indicators of temperature stress in the brook trout (Salvelinus fontinalis). Canadian Journal of Fisheries and Aquatic Sciences 60, 460-470.

Thomsen, M.T., Wang, T., Milsom, W.K., Bayley, M., 2017. Lactate provides a strong pH-independent ventilatory signal in the facultative air-breathing teleost Pangasianodon hypophthalmus. Scientific Reports 7, 6378.

Thorne, M.A.S., Burns, G., Fraser, K.P.P., Hillyard, G., Clark, M.S., 2010. Transcription profiling of acute temperature stress in the Antarctic plunderfish Harpagifer antarcticus. Marine Genomics 3, 35-44.

Van Meer, G., Voelker, D.R., Feigenson, G.W., 2008. Membrane lipids: where they are and how they behave. Nature Reviews Molecular Cell Biology 9, 112-124.

Vatanparast, M., Ahmed, S., Lee, D. H., Hwang, S.H., Hammock, B., Kim, Y., 2020. EpOMEs act as immune suppressors in a lepidopteran insect, Spodoptera exigua. Scientific Reports 10, 20183.

Vetter, W., Darwisch, V., Lehnert, K., 2020. Erucic acid in Brassicaceae and salmon - An evaluation of the new proposed limits of erucic acid in food. NFS Journal 19, 9-15. 
Wang, Q.J., Ju, X., Chen, Y.K., Dong, X.Q., Luo, S., Liu, H.J., Zhang, D.M., 2016. Effects of L-carnitine against $\mathrm{H}_{2} \mathrm{O}_{2}$-induced oxidative stress in grass carp ovary cells (Ctenopharyngodon idellus). Fish Physiology and Biochemistry 42, 845-857.

Windisch, H.S., Frickenhaus, S., John, U., Knust, R., P?Rtner, H.O., Lucassen, M., 2014. Stress response or beneficial temperature acclimation: transcriptomic signatures in Antarctic fish (Pachycara brachycephalum). Molecular Ecology 23, 3469-3482.

Xia, J., Peng, J. L., Fu, S.J., 2017. The Behavioral Response of Juvenile Brachymystax lenok tsinlingensis to Heating Stress. Journal of Chongqing Normal University (Natural Science) (in Chinese with English abstract).

Xu, G.F., Wang, Y.Y., Han, Y., Xiang, L.I., Bo, M.A., Liu, Y., Mou, Z.B., 2014. Effect of locomotion and feeding on metabolic mode of juvenile lenok, Brachymystax lenok (Pallas) under different water temperatures. Chinese Journal of Applied Ecology. (in Chinese with English abstract)

Yang, P.Y., Klein, R.D., Chan, D., Felix, E., Madden, T., Shureiqi, I., Cheo, X.X., Dannenberg, A.J., Newman, R.A., 2006. LC/MS/MS based determination of tissue inflammation profiles: Simultaneous determination of COX and LOX derived bioactive lipids. Prostaglandins \& Other Lipid Mediators 79, 187-187.

Yeo, S.R., Kim, K.Y., 2014. Global warming, low-frequency variability, and biennial oscillation: an attempt to understand the physical mechanisms driving major ENSO events. Climate Dynamics 43, 771-786.

Zarini, S., Hankin, J.A., Murphy, R.C., Gijon, M.A., 2014. Lysophospholipid acyltransferases and eicosanoid biosynthesis in zebrafish myeloid cells. Prostaglandins \& Other Lipid Mediators 113, 52-61.

Zhang, W., Song, Y., Chai, T.T., Liao, G.Q., Zhang, L., Jia, Q., Qian, Y.Z., Qiu, J., 2020. Lipidomics perturbations in the brain of adult zebrafish (Danio rerio) after exposure to chiral ibuprofen. Science of the Total Environment 713, 136565.

\section{Figures}




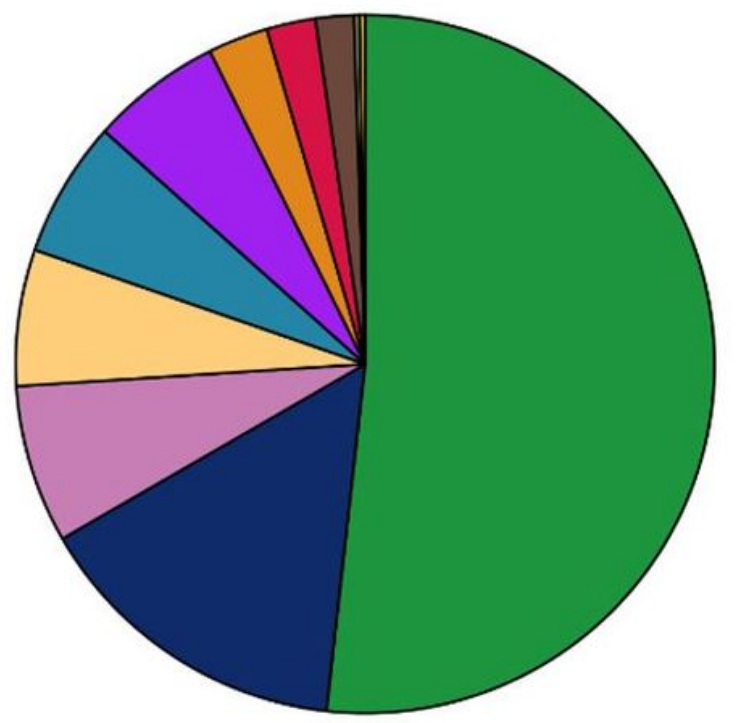

Superclass

Undefined $51.768 \%$

- Organic acids and derivatives $14.899 \%$

$\square$ Lipids and lipid-like molecules $7.323 \%$

"Nucleosides, nucleotides, and analogues" $6.313 \%$

Organoheterocyclic compounds $6.313 \%$

$\square$ Organic oxygen compounds $6.061 \%$

ㅁ Organic nitrogen compounds $2.778 \%$

Benzenoids $2.273 \%$

$\square$ Phenylpropanoids and polyketides $1.768 \%$

口 "Nucleosides, nucleotides, and analogues/Organoheterocyclic compounds" $0.253 \%$

$\square$ Organosulfur compounds $0.253 \%$

\section{Figure 1}

The percentage of identified metabolites account for Chemical Taxonomy
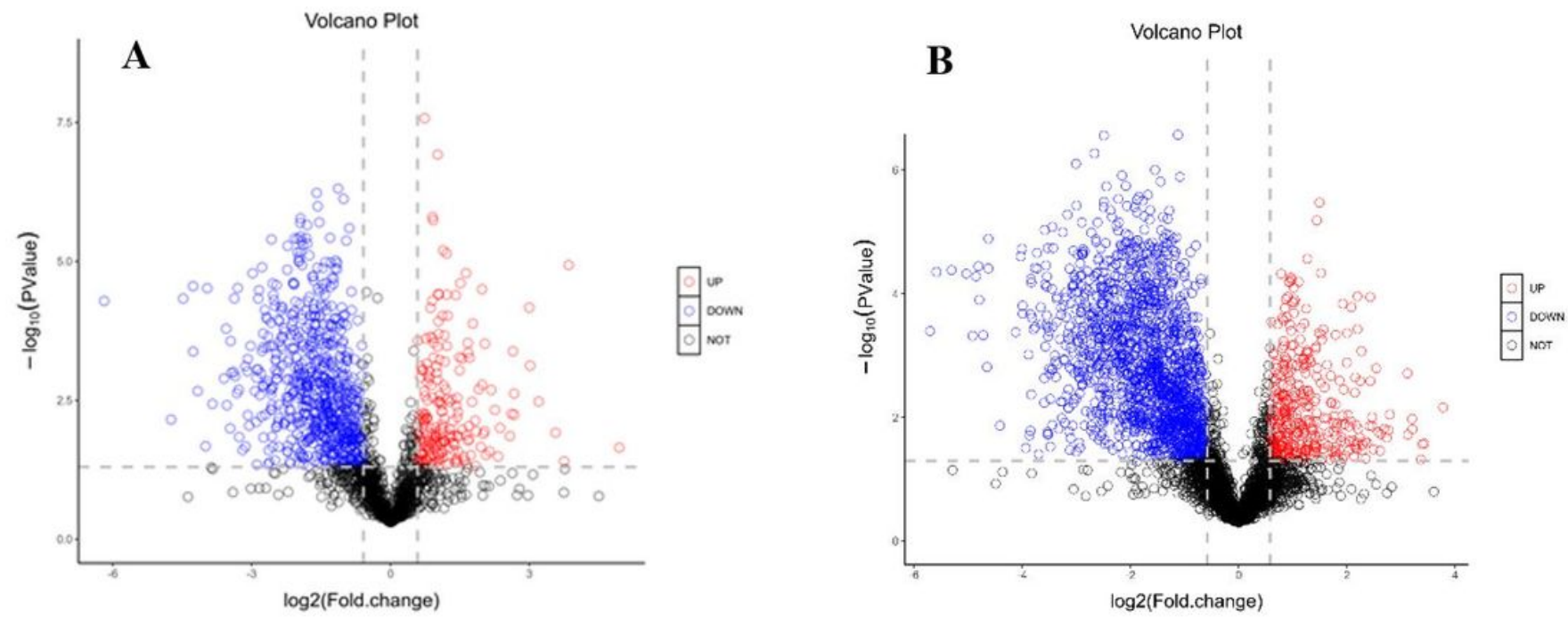

\section{Figure 2}

Volcano plots of metabolites in lenok after heat stress with $\mathrm{FC}>1.5$ or $\mathrm{FC}<0.67$, and $\mathrm{p}<0.05$ in positive (A) and negative (B) ion mode. Color denotes the abundance of metabolites from the highest (red) to the lowest (blue). 

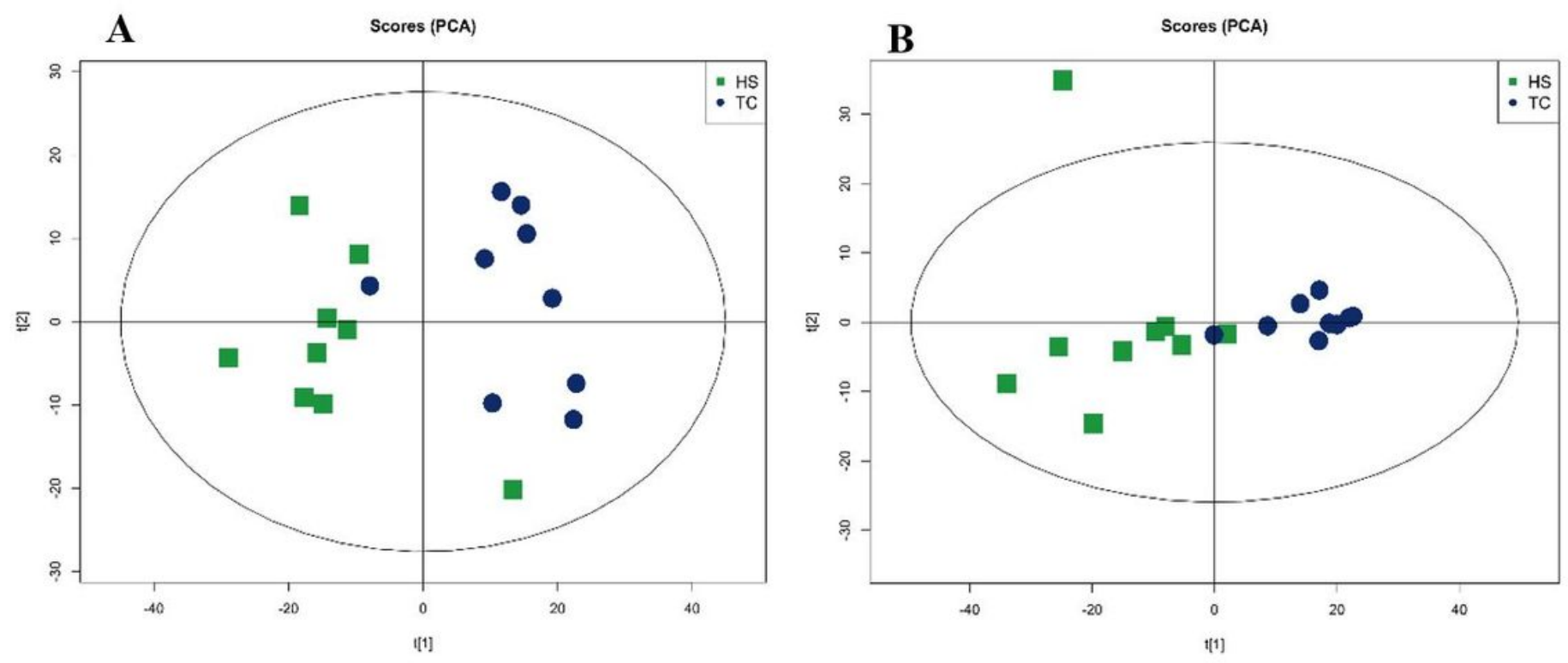

Figure 3

PCA score plots in positive (A) and negative (B) ion mode, showing separation between the control (blue) and heat stress (green) treatment. Each dot represents one intestinal sample from each treatment. 

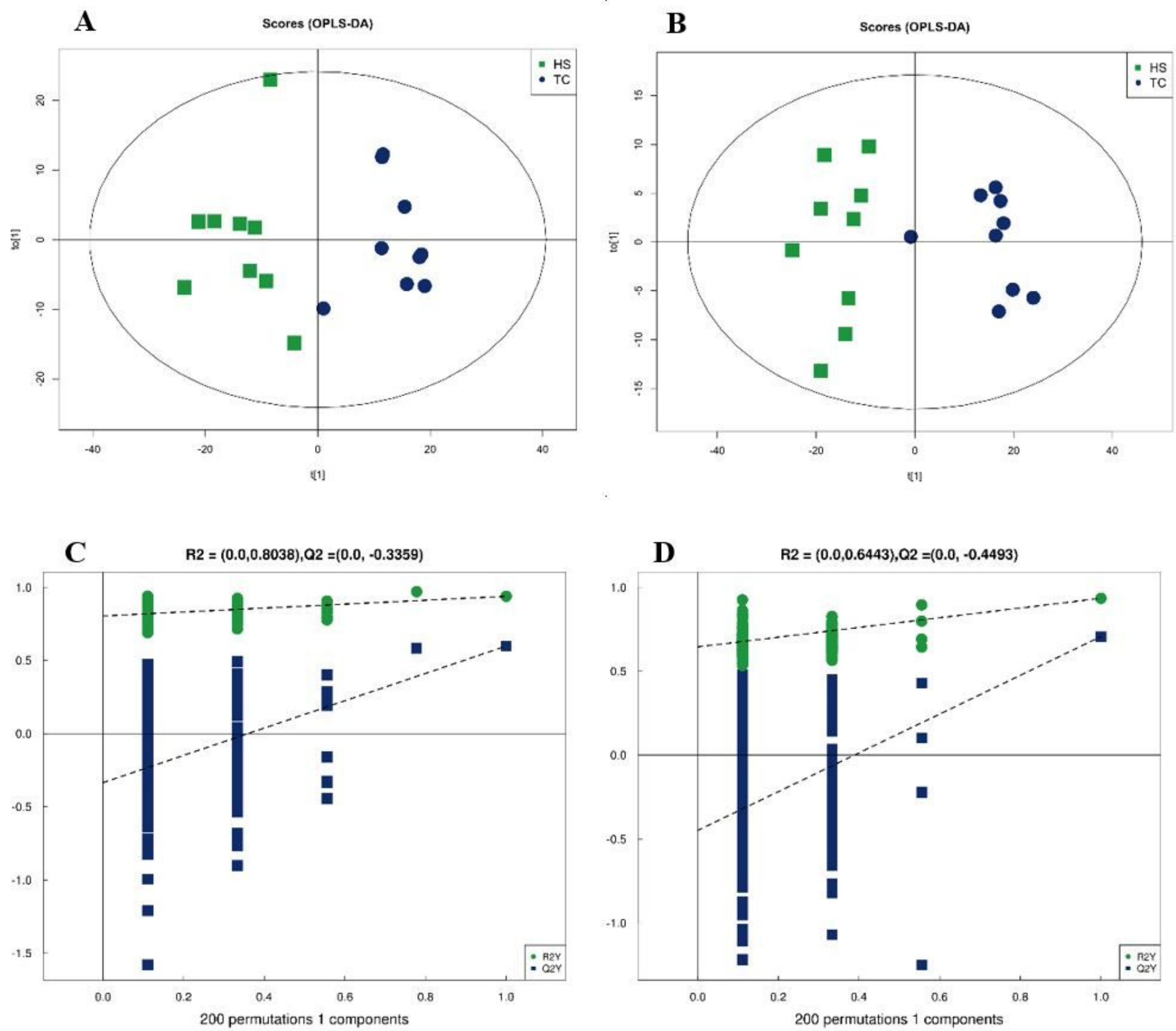

Figure 4

OPLS-DA for discriminating the intestinal metabolite profiles in control (blue) and heat stress (green) treatments, with each dot representing one intestinal sample from each treatment in positive $(A)$ and negative ion mode $(B)$, and the corresponding permutation test ( $C$, positive ion mode) and ( $D$, negative ion mode). 

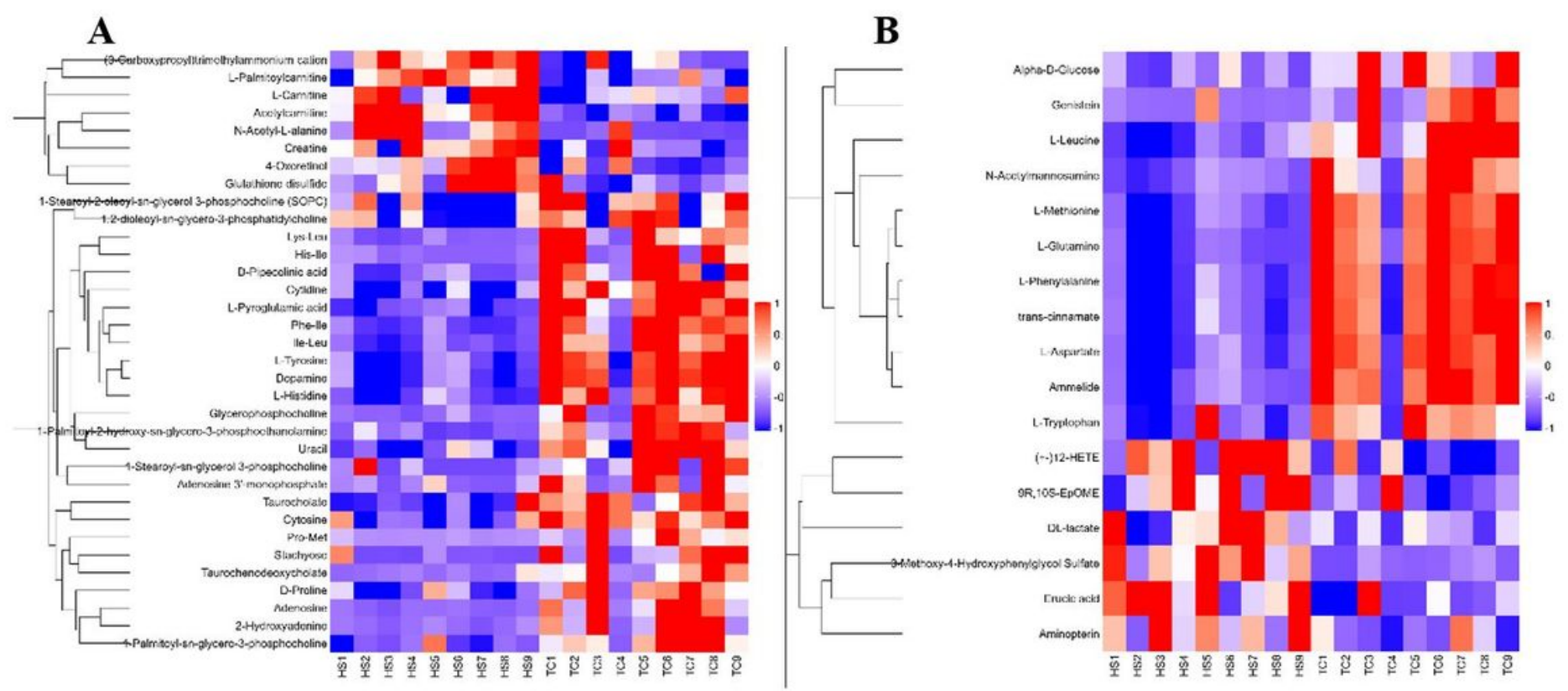

\section{Figure 5}

Hierarchical clustering analysis of the significantly different metabolites in response to heat stress of positive $(A)$ and negative $(B)$ ion mode. Color denotes the abundance of metabolites, from the highest (red) to the lowest (blue).

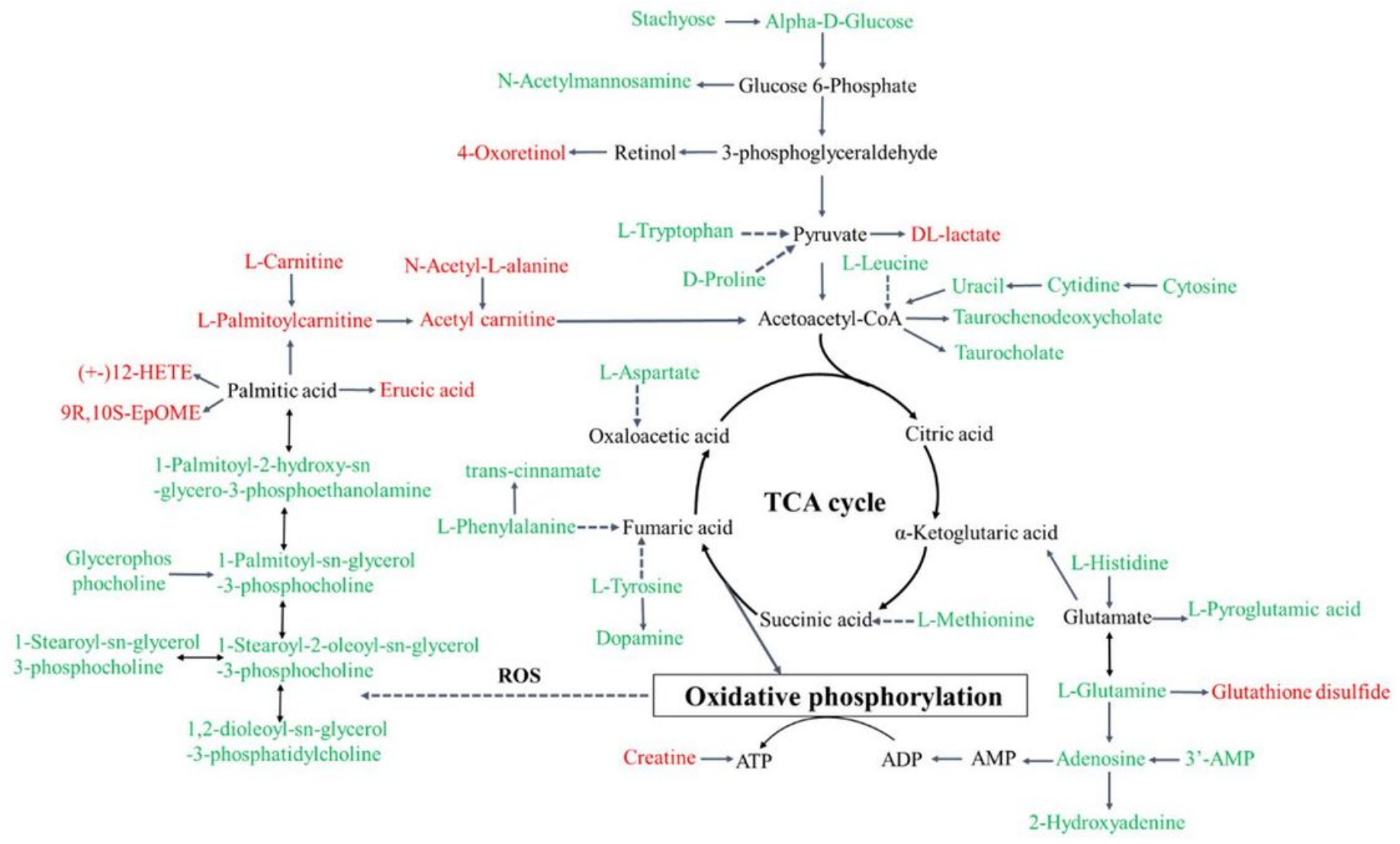

Figure 6 
The hypothetical framework based on the changed metabolites of heat treated lenok compared with control group. The metabolites are colored according to the type of change after the heat stress (black, no change; red, up-regulation; green, down-regulation).

\section{Supplementary Files}

This is a list of supplementary files associated with this preprint. Click to download.

- SupplementaryMaterial.docx 